\title{
Estimativas volumétricas para um povoamento adensado de Eucalyptus sp. em regime de curta rotação
}

\author{
Jéssica Silva Nunes ${ }^{1}$, Thelma Shirlen Soares ${ }^{1}$ \\ ${ }^{1}$ Universidade Federal de Goiás, Regional Jataí, Jataí, Goiás, Brasil. E-mail: jessicaanunees@ hotmail.com, thelmasoares@ufg.br
}

Recebido:15/05/2017; Aceito: 29/11/2017

\section{RESUMO}

Este estudo teve como objetivo estabelecer equações volumétricas, fatores de forma, de empilhamento e de cubicação para um povoamento de eucalipto com 24 meses de idade, implantado em regime de curta rotação, sob diferentes espaçamentos $(3,0 \mathrm{~m} \times 0,5 \mathrm{~m} ; 3,0 \mathrm{~m} \times 1,0 \mathrm{~m} ; 3,0 \mathrm{~m} \times 1,5 \mathrm{~m} ; 3,0 \mathrm{~m} \times 2,0 \mathrm{~m} ; 3,0 \mathrm{~m} \times 2,5 \mathrm{~m}$ e $3,0 \mathrm{~m} \times$ $3,0 \mathrm{~m}$ ), em Jataí-GO. Para o ajuste volumétrico as árvores foram cubadas pelo método de Smalian, sendo avaliadas seis equações para cada espaçamento. Após a seleção das equações realizou-se o teste de identidade de modelos. Também foram obtidos, para cada espaçamento, os fatores de forma, empilhamento e cubicação. A equação oriunda do modelo de Spurr foi selecionada para os espaçamentos 3,0 m x 0,5 m; 3,0 m x 1,5 m e 3,0 m x 2,0 m. Para os espaçamentos 3,0 m x 1,0 m e 3,0 m x 3,0 m a equação originada pelo modelo de Schumacher-Hall foi a selecionada. Já no espaçamento $3,0 \mathrm{~m}$ x 2,5 m a equação selecionada foi a gerada pelo modelo de Stoate. Pelo teste de identidade de modelos, verificou-se que apenas nas junções dos espaçamentos $3,0 \mathrm{~m}$ x $0,5 \mathrm{~m}$ e 3,0 m x 2,0 m pode-se utilizar de um modelo reduzido sendo este: $V=0,006661+0,000036$ dap ${ }^{2} h$. Os fatores de forma variaram entre 0,48 e 0,56 , enquanto que os fatores de empilhamento entre 1,44 a 2,05 e os fatores de cubicação de 0,57 a 0,70 .

Palavras-chave: fatores de forma, fatores de empilhamento, teste de identidade de modelos

\section{Volumetric estimates for a hight stacked stand of Eucalyptus sp. in short rotation system}

\begin{abstract}
This study aimed to establish volumetric equations, factors, piling and cubication for eucalyptus stand with 24 months of age, deployed on short rotation system under different spacing $(3.0 \mathrm{~m} \times 0.5 \mathrm{~m} ; 3,0 \mathrm{~m} \times 1.0 \mathrm{~m}, 3.0 \mathrm{~m} \mathrm{x}$ $1.5 \mathrm{~m}, 3.0 \mathrm{~m} \times 2.0 \mathrm{~m}, 3.0 \mathrm{~m} \times 2.5 \mathrm{~m}$ and $3.0 \mathrm{~m} \times 3.0 \mathrm{~m}$ ) in Jataí - State of Goiás, Brazil. For volume adjustment the trees were cubed by Smalian method being evaluated six equations for each spacing. After selecting the equations there was the model identity test. Were also obtained for each spacing, the form factors, stacking and cubication. The equation derived from the Spurr model was selected for the spacing $3.0 \mathrm{~m} \times 0.5 \mathrm{~m} ; 3.0 \mathrm{~m} \times 1.5 \mathrm{~m}$ x $2.0 \mathrm{~m}$ and $3.0 \mathrm{~m}$. The Schumacher-Hall model was selected for spacing $3.0 \mathrm{~m}$ x $1.0 \mathrm{~m}$ and $3.0 \mathrm{~m}$ x $3.0 \mathrm{~m}$. Already spaced $3.0 \mathrm{~m}$ x $2.5 \mathrm{~m}$ selected equation was generated by Stoate model. From the model identity test, it was found that only the junctions of spacings $3.0 \mathrm{~m} \mathrm{x} 0.5 \mathrm{~m}$ and $3.0 \mathrm{~m} \mathrm{x} 2.0 \mathrm{~m}$ can be used a reduced model, which is: $V=0.006661+0.000036 d_{a p}^{2} h$. The form factors varied between 0.48 and 0.56 , while the piling factor between 1.44 and 2.05 and the cubication factors from 0.57 to 0.70 .
\end{abstract}

Key words: form factors, stack factors, models identity tests. 


\section{Introdução}

Estimar o volume de madeira é de suma importância no controle de estoque, no controle do crescimento, no planejamento da produção, na elaboração de plano de manejo, na comercialização, no transporte de produtos florestais e na adequação dos equipamentos processadores de madeira (picadores, serras, etc.), facilitando ainda o conhecimento do potencial florestal em uma região (BARROS et al., 2008).

Portanto, conhecer o volume de madeira de uma floresta é de extrema relevância para fundamentar os processos de gestão de empreendimentos florestais.

Entre os métodos empregados para a estimativa da produção volumétrica de um povoamento florestal, estão os modelos volumétricos, os fatores de forma, os quocientes de forma, os fatores de empilhamento e os fatores de cubicação, sendo que a utilização de um ou outro método depende da facilidade de obtenção dos dados e da precisão requerida.

Muitos estudos foram desenvolvidos analisando os diferentes métodos de estimação volumétrica de povoamentos florestais, dentre eles os trabalhos desenvolvidos por Barros et al. (2008), Miguel et al. (2010), Rocha et al. (2010), Azevedo et al. (2011).

Entretanto, considerando que o crescimento florestal é influenciado por inúmeros fatores, tais como luminosidade, disponibilidade de nutrientes, densidade de plantio, genética, tipo de solo, clima, dentre outros
(PACHECO et al., 2015), torna-se necessário que os métodos empregados para as estimativas volumétricas sejam testados para cada condição específica de desenvolvimento da espécie e condições de manejo.

Neste contexto, foi desenvolvido este estudo cujo objetivo foi estabelecer equações volumétricas e fatores de forma, empilhamento e cubicação para um povoamento de eucalipto em regime de curta rotação sob diferentes espaçamentos.

\section{Material e Métodos}

Foram utilizados dados do clone 1277 (E. grandis $\mathrm{x}$ E. camaldulensis) aos 26 meses de idade, implantado em Jataí-GO, em seis espaçamentos de plantio (3,0 $\mathrm{m} \mathrm{x}$ $0,5 \mathrm{~m}, 3,0 \mathrm{~m} \times 1,0 \mathrm{~m} ; 3,0 \mathrm{~m} \times 1,5 \mathrm{~m} ; 3,0 \mathrm{~m} \times 2,0 \mathrm{~m} ; 3,0$ $\mathrm{m} \times 2,5 \mathrm{~m} \mathrm{e} 3,0 \mathrm{~m} \times 3,0 \mathrm{~m})$.

Para a obtenção das relações volumétricas foram cubadas rigorosamente, pelo método de Smalian, 45 árvores em cada espaçamento. As medidas foram obtidas nas alturas 0,$1 ; 0,5 ; 1,0 ; 2,0 ; 4,0 ; 6,0 \mathrm{~m}$, e assim sucessivamente até o diâmetro de $5 \mathrm{~cm}$ de fuste com casca. Foram avaliados, seis modelos clássicos de simples e dupla entrada, ou seja, com uma e duas variáveis independentes, respectivamente, os quais estão descritos na Tabela 1.

A análise de variância utilizada para os modelos lineares está apresentada na Tabela 2.

Tabela 1. Modelos testados para estimativa do volume total de árvores individuais*

\begin{tabular}{|c|c|c|}
\hline Modelo & Autor & Modelo \\
\hline 1 & Hohenadl-Krenn & $V=\beta_{0}+\beta_{1} d a p+\beta_{2} d a p^{2}+\varepsilon_{i}$ \\
\hline 2 & Husch & $\ln (V)=\beta_{0}+\beta_{I} \ln ($ dap $)+\varepsilon_{i}$ \\
\hline 3 & Brenac & $\ln (V)=\beta_{0}+\beta_{1} \ln ($ dap $)+\beta_{2} d a p^{-1}+\varepsilon_{i}$ \\
\hline 4 & Spurr & $V=\beta_{0}+\beta_{l} \operatorname{dap}^{2} h+\varepsilon_{i}$ \\
\hline 5 & Stoate & $V=\beta_{0}+\beta_{1} d a p^{2}+\beta_{2} d a p^{2} h+\beta_{3}{ }^{2} h+\varepsilon_{i}$ \\
\hline 6 & Schumacher-Hall & $V=\beta_{0} d a p^{\beta 1} h^{\beta 2}+\varepsilon_{i}$ \\
\hline
\end{tabular}

* Em que: $\ln =$ logaritmo neperiano; $\mathrm{v}=$ volume $\left(\mathrm{m}^{3}\right) ;$ dap $=$ diâmetro medido a $1,3 \mathrm{~m}$ do solo $(\mathrm{cm}) ; \mathrm{h}=$ altura do fuste $(\mathrm{m}) ; \beta_{0}, \beta_{1}, \beta_{2}$ e $\beta_{3}=$ parâmetros a serem estimados; $\varepsilon_{\mathrm{i}}=$ erro aleatório, $\varepsilon \mathrm{i} \sim \mathrm{NID}\left(0, \sigma^{2}\right)$.

Tabela 2. Análise de variância para o teste de identidade do modelo linear.

\begin{tabular}{ccccc}
\hline Fonte de Variação & GL & SQ & QM & Fc \\
\hline Modelo completo & $h \times p$ & $\beta^{\prime} X^{\prime} Y$ & & \\
Modelo reduzido & $p$ & $\theta^{\prime} Z^{\prime} Y$ & $S Q_{d \text { ijerenşa }}$ & $\frac{S Q_{d j \text { jerenşa }}}{(h-1) \times p}$ \\
Diferença & $(h-1) x p$ & $S Q_{\text {completo }}-S Q_{\text {reduzido }}$ & $\frac{S Q_{\text {residuo }}}{n-(h \times p)}$ & $\frac{Q M_{\text {dijerença }}}{Q M_{\text {residuo }}}$ \\
Resíduo & $n-(h x p)$ & $S Q_{\text {total }}-S Q_{\text {completo }}$ &
\end{tabular}

Em que: $h=$ número de espaçamentos; $p=$ número de parâmetros do modelo; $n=$ número de observações; $G L=$ graus de liberdade; $S Q=$ soma de quadrados; $Q M=$ quadrado médio; $\mathrm{Fc}=$ estatística $\mathrm{F}$. 
A seleção do melhor modelo para a obtenção das estimativas dos volumes foi feita com base nos seguintes critérios: análise de variância $(F)$, coeficiente de determinação ajustado ( $R^{2} a j$ ), erro padrão da estimativa $\left(\mathrm{S}_{\mathrm{yx}} \%\right)$ e análise gráfica dos resíduos, conforme Thomas et al. (2006). Para fins de comparação dos modelos logarítmicos com os não logarítmicos foi recalculado o erro padrão da estimativa por meio do fator de correção de Meyer (FM):

$$
\mathrm{FM}=\mathrm{e}^{0,5 \mathrm{QM}_{\text {erro }}}
$$

em que: $\mathrm{e}=$ base do logaritmo natural; $\mathrm{QM}_{\text {erro }}=$ quadrado médio do erro.

Após o ajuste das equações volumétricas, empregouse o teste de identidade de modelos (TIM) para verificar a possibilidade de uma única equação representar a relação volumétrica para os tratamentos agrupados, sendo este aplicado aos modelos lineares e não-lineares de forma distinta. Considerando que no presente estudo serão selecionadas uma equação para cada espaçamento, a aplicação do teste de identidade permite verificar se as equações são estatisticamente iguais e se uma única equação pode ser obtida para representar todas elas sem perda de informação para a inferência. De acordo com Queiroz et al. (2008), o TIM rejeita ou aceita uma determinada hipótese por meio da redução da soma de quadrados da regressão e uma de suas principais características é permitir a avaliação do comportamento de uma ou mais variáveis em condições distintas.

Para os modelos lineares foi empregado o teste de identidade descrito por Graybill (1976), baseado no teste de F, a partir da redução da soma de quadrados. Essa técnica também foi utilizada por Camolesi et al. (2010), Regazzi e Silva (2010), Kurchaidt et al. (2014) e Nicoletti et al. (2016), entre outros. No TIM para os modelos não-lineares, empregou-se o teste da razão de verossimilhanças, com aproximação dada pela estatística qui-quadrado $\left(X_{\mathrm{c}}^{2}\right)$ :

$$
\mathrm{X}_{\mathrm{C}}^{2}=-\mathrm{n} \times \ln \left(\frac{\mathrm{SQR}_{\text {completo }}}{\mathrm{SQR}_{\text {red uzido }}}\right)
$$

em que: $X_{c}^{2}=$ estatística qui-quadrado; $\ln =$ logaritmo neperiano; $\mathrm{SQR}_{\text {reduzido }}=$ soma de quadrado dos resíduos do modelo reduzido; $\mathrm{e}, \mathrm{SQR}_{\text {completo }}=$ soma de quadrado dos resíduos do modelo completo.

Para testar as hipóteses acima é comparado o valor de $X^{2}$ calculado com o valor tabelado em função do nível $\alpha$ de probabilidade e graus de liberdade entre a diferença do número de coeficientes do modelo completo e do modelo reduzido. Se o valor de $X^{2}$ calculado for maior que o valor crítico fornecido pela tabela, rejeita-se a hipótese de nulidade $\left(\mathrm{H}_{0}\right)$, não podendo assim utilizar o modelo reduzido. Nesse caso, as hipóteses testadas são: $\mathrm{H}_{0}=\mathrm{o}$ modelo reduzido ajustado para todos os volumes em questão é idêntico aos modelos completos ajustados para cada volume e $\mathrm{H}_{1}$ $=$ rejeita-se $\mathrm{H}_{0}$.

Além do uso das equações volumétricas para obter o volume de madeira em cada espaçamento de plantio, foram obtidos fatores de forma, empilhamento $\mathrm{e}$ cubicação.

$\mathrm{O}$ fator de forma (f) foi calculado pela razão entre o volume do cilindro e o volume real da árvore:

$$
f=\frac{V_{c}}{V_{r}}
$$

em que: $f=$ fator de forma; $V_{c}=$ volume do cilindro; e, $V_{r}=$ volume real da árvore determinado pela cubagem.

Para obtenção dos fatores de empilhamento e cubicação, foram obtidos 30 toretes de $2 \mathrm{~m}$ de comprimento para cada parcela, os quais foram cubados pelo método de Smalian. As pilhas foram organizadas, manualmente, para cada espaçamento estudado, sendo considerados no processo de empilhamento, toras com diâmetros variados, independentemente da posição que esta ocupava na árvore.

O volume da madeira empilhada foi calculado a partir das dimensões das pilhas:

$$
V s t=L \times C \times H
$$

em que: $V s t=$ volume da pilha ( $\mathrm{m}$ estere); $L=$ largura da pilha (m); $C=$ comprimento da pilha (m); e, $H=$ altura da pilha (m).

O fator de empilhamento (fe) é definido pela razão entre o volume empilhado e o volume sólido da pilha de madeira:

$$
f e=\frac{\text { Volume empilhado }}{\text { Volume sólido }}
$$

O fator de cubicação (fc) usado na conversão de madeira empilhada em volume de madeira maciça foi obtido pela razão entre o volume sólido e o volume empilhado, sendo:

$$
f_{c}=\frac{\text { Volume sólido }}{\text { Volume empilhado }}
$$

\section{Resultados e Discussão \\ Modelos volumétricos}

Para o espaçamento $3,0 \mathrm{~m} \times 0,5 \mathrm{~m}$ os resultados pertinentes aos ajustes, com os respectivos parâmetros estatísticos de precisão e distribuição gráfica dos resíduos estão apresentados na Tabela 3 e Figura 1, respectivamente.

Para os modelos de Hohenadl-Kren, Husch, Stoate e Schumacher-Hall, é notória uma clara tendência de subestimativa para as árvores de menor diâmetro, enquanto que o modelo de Brenac apresentou ligeira tendência em superestimativa para as árvores de dap entre 6 e $8 \mathrm{~cm}$ (Figura 1). 
Tabela 3. Parâmetros estimados e medidas de precisão das equações volumétricas ajustadas para o híbrido E. grandis x E. camaldulensis (clone 1277), implantado no espaçamento 3,0 m x 0,5 m, em Jataí-GO

\begin{tabular}{cccccccc}
\hline Equação & $\beta_{0}$ & $\beta_{1}$ & $\beta_{2}$ & $\beta_{3}$ & $\mathrm{R}^{2}$ aj. $(\%)$ & $\mathrm{S}_{\mathrm{yx}}(\%)$ & $\mathrm{F}$ \\
\hline Hohenadl-Krenn & 0,01281 & $-0,00347$ & 0,00078 & & 97,2 & $\pm 6,1$ & 9,8 \\
Husch & $-7,48831$ & 1,99745 & & & 96,8 & $\pm 6,3$ & 25,9 \\
Brenac & $-11,6979$ & 3,3646 & 10,6566 & & 97,5 & $\pm 6,3$ & 15,0 \\
Spurr* & 0,00603 & 0,00004 & & & 97,7 & $\pm 5,5$ & 21,3 \\
Stoate & $-0,00965$ & 0,00038 & 0,00001 & 0,00100 & 98,5 & $\pm 4,6$ & 14,8 \\
Schumacher-Hall & 0,00019 & 1,88417 & 0,50151 & & 98,5 & $\pm 4,4$ & 15,7 \\
\hline
\end{tabular}

Em que: $\beta_{0}, \beta_{1}, \beta_{2} \beta_{3}=$ coeficientes da regressão, $\mathrm{R}^{2}$ aj. $(\%)=$ coeficiente de determinação ajustado, $\mathrm{S}_{\mathrm{yx}}(\%)=$ erro em porcentagem, $\mathrm{F}=$ estatística $\mathrm{F}$ de Fischer, $*=$ modelo selecionado.
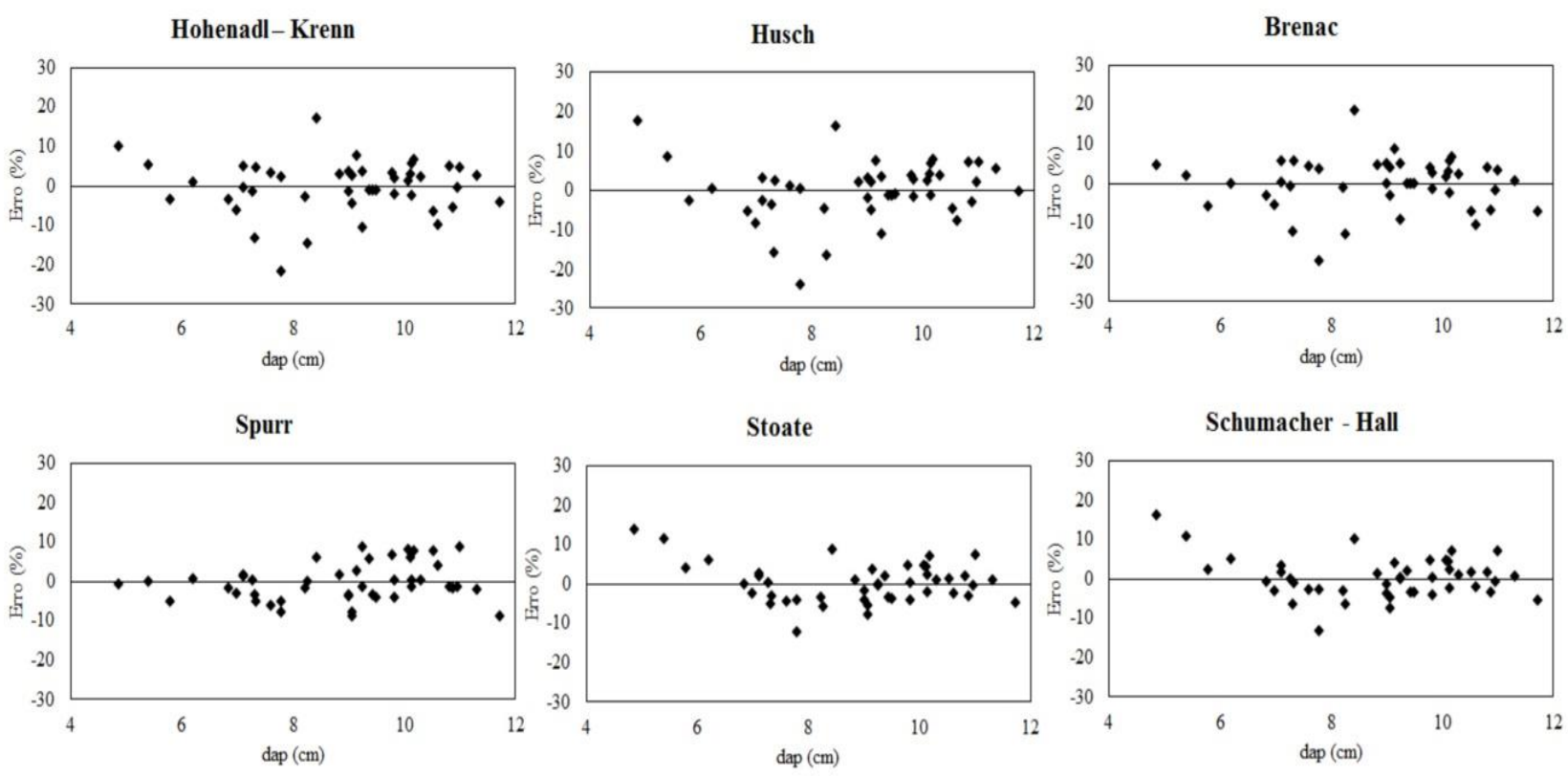

Figura 1. Resíduos em função do diâmetro para as equações volumétricas ajustadas para o híbrido E. grandis x E. camaldulensis (clone 1277) implantado no espaçamento 3,0 m x 0,5 m em Jataí-GO.

A equação que melhor se ajustou ao conjunto de dados neste espaçamento foi a de Spurr, com um coeficiente de determinação de $97,7 \%$ e um erro de $5,5 \%$, sendo que o critério de desempate para seleção desta equação foi a distribuição gráfica dos resíduos.

Os resultados dos ajustes, com os respectivos parâmetros estatísticos de precisão e distribuição gráfica dos resíduos para o espaçamento $3,0 \mathrm{~m} \times 1,0 \mathrm{~m}$ estão apresentados na Tabela 4 e Figura 2 respectivamente.

Para os modelos de Hohenadl-Kren, Husch, Stoate e Schumacher-Hall, é notória uma clara tendência de subestimativa para as árvores de menor diâmetro, enquanto que o modelo de Brenac apresentou ligeira tendência em superestimativa para as árvores de dap entre 6 e $8 \mathrm{~cm}$ (Figura 1).

A equação que melhor se ajustou ao conjunto de dados neste espaçamento foi a de Spurr, com um coeficiente de determinação de $97,7 \%$ e um erro de
5,5\%, sendo que o critério de desempate para seleção desta equação foi a distribuição gráfica dos resíduos.

Os resultados dos ajustes, com os respectivos parâmetros estatísticos de precisão e distribuição gráfica dos resíduos para o espaçamento $3,0 \mathrm{~m} \mathrm{x} 1,0 \mathrm{~m}$ estão apresentados na Tabela 4 e Figura 2 respectivamente.

Pela dispersão gráfica dos resíduos, verifica-se que as equações de Hohenadl-Krenn, Husch, Brenac, Stoate e Schumacher-Hall apresentaram tendência de superestimativa dos volumes para as árvores com diâmetro entre 10 e $12 \mathrm{~cm}$, sendo que apenas a equação de Spurr apresentou distribuição de resíduos livre de tendências em comparação às demais equações.

Para este espaçamento, Spurr foi a equação escolhida por possuir um coeficiente de determinação de $94,1 \%$ e erro padrão da estimativa de $6,1 \%$, sendo a distribuição gráfica dos resíduos decisiva para a seleção desta equação. 
Tabela 4. Parâmetros estimados e medidas de precisão das equações volumétricas ajustadas para o híbrido $E$. grandis x $E$. camaldulensis (clone 1277), implantado no espaçamento 3,0 m x 1,0 m, em Jataí-GO.

\begin{tabular}{cccccccc}
\hline Equação & $\beta_{0}$ & $\beta_{1}$ & $\beta_{2}$ & $\beta_{3}$ & $\mathrm{R}^{2}$ aj. $(\%)$ & $\mathrm{S}_{\mathrm{yx}}(\%)$ & $\mathrm{F}$ \\
\hline Hohenadl-Krenn & $-0,02602$ & 0,00497 & 0,00030 & & 97,9 & $\pm 5,5$ & 25,9 \\
Husch & $-7,92364$ & 2,16444 & & & 98,1 & $\pm 6,1$ & 8,4 \\
Brenac & $-5,13749$ & 1,30633 & $-7,92915$ & & 98,5 & $\pm 5,3$ & 15,0 \\
Spurr & 0,00802 & 0,00003 & & & 98,7 & $\pm 4,1$ & 21,3 \\
Stoate & $-0,00705$ & 0,00015 & 0,00002 & 0,00119 & 98,8 & $\pm 4,0$ & 18,4 \\
Schumacher-Hall* & 0,00011 & 1,69694 & 0,85720 & & 98,9 & $\pm 3,6$ & 10,7 \\
\hline
\end{tabular}

Em que: $\beta_{0}, \beta_{1}, \beta_{2} \beta_{3}=$ coeficientes da regressão, $\mathrm{R}^{2}$ aj. $(\%)=$ coeficiente de determinação ajustado, $\mathrm{S}_{\mathrm{yx}}(\%)=$ erro em porcentagem, $\mathrm{F}=$ estatística $\mathrm{F}$ de Fischer, $*=$ modelo selecionado.
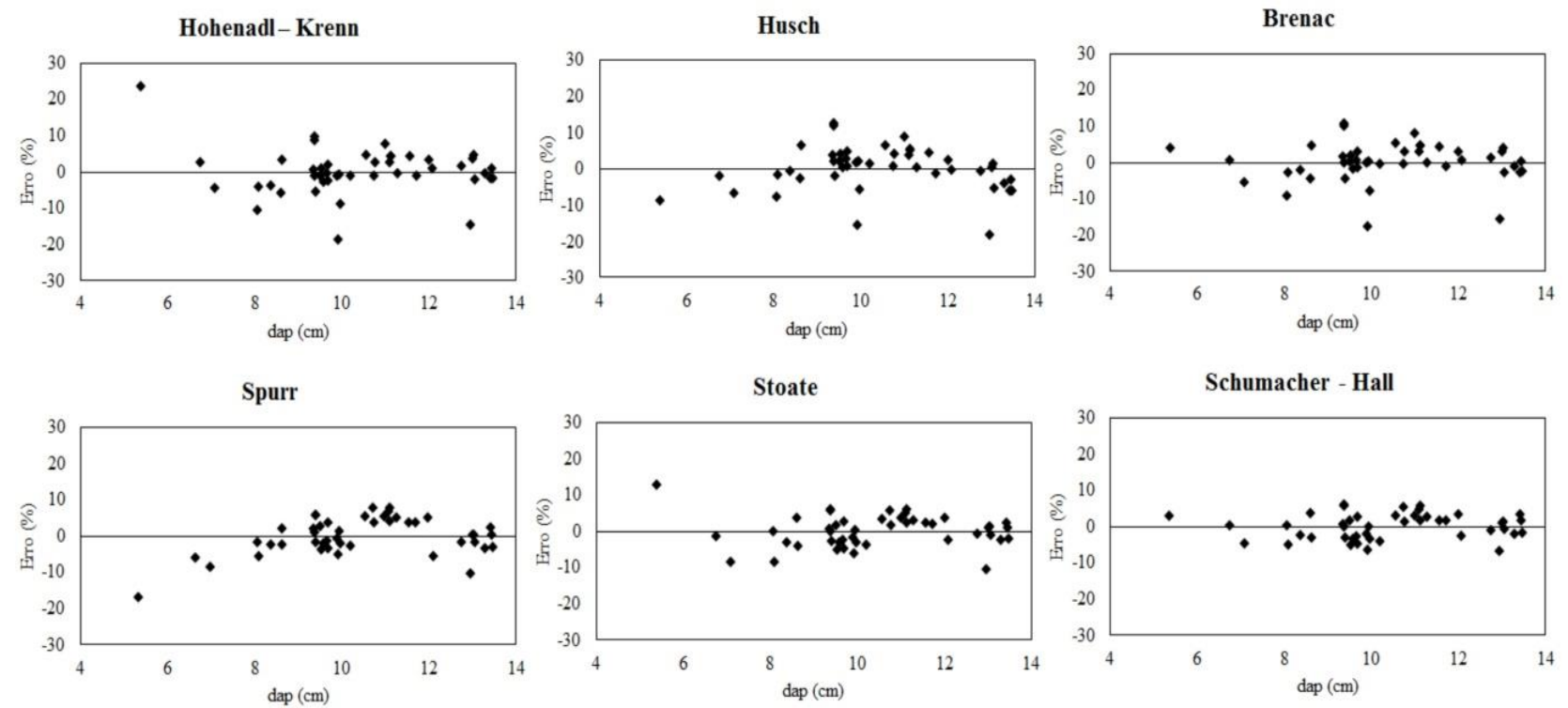

Figura 2. Resíduos em função do diâmetro para as equações volumétricas ajustadas para o híbrido E. grandis x E. camaldulensis (clone 1277) implantado no espaçamento 3,0 m x 1,0 m em Jataí-GO.

Na Figura 4 e Tabela 6 encontram-se, para o espaçamento $3,0 \mathrm{~m}$ x 2,0 $\mathrm{m}$, os resultados dos parâmetros e da estatística para os modelos ajustados. Spurr com um coeficiente de determinação de $91,5 \%$ e erro padrão da estimativa de $5,8 \%$ foi também a equação escolhida para o espaçamento $3,0 \mathrm{~m}$ x 2,0 m, também escolhida em função da melhor distribuição gráfica dos resíduos.

As medidas de precisão, os parâmetros das equações e a distribuição gráfica dos resíduos estão descritos na Tabela 7 e Figura 5.

$\mathrm{Na}$ análise da distribuição gráfica dos resíduos, verifica-se que apenas a equação de Stoate apresentou boa distribuição com estimativas livre de tendência em comparação às demais equações. Portanto, Stoate foi a equação selecionada para o espaçamento $3,0 \mathrm{~m}$ x 2,5 m. Os valores para o coeficiente de determinação e erro padrão da estimativa foram de $93,8 \%$ e $4,3 \%$ respectivamente.

Melo et al. (2013), estudando modelos volumétricos para povoamentos de Pinus caribaeae var. hondurensis com 10 anos de idade nos espaçamentos 3,0 m x 3,0 m, constataram que o modelo de Stoate foi o mais eficiente na estimativa dos volumes total e comercial com casca.

Os ajustes do modelo e os parâmetros de precisão estão descritos na Tabela 8 e a distribuição gráfica dos resíduos na Figura 6. A equação de Schumacher-Hall mostrou-se superior a todas as demais na distribuição gráfica de resíduos, apresentando uma distribuição mais homogênea dos resíduos. Sendo assim, para o espaçamento 3,0 m x 3,0 m, selecionou-se a equação de Schumacher-Hall, a qual gerou valores para o coeficiente de determinação de $93,4 \%$ e erro padrão da estimativa de $5,6 \%$.

Thomas et al. (2006) trabalhando com comparações de equações volumétricas ajustadas com dados de cubagem e análise de tronco para Pinus taeda L., variando de 8 a 28 anos de idade, com diferentes densidades de plantios e classes diamétricas, também selecionaram Schumacher-Hall como a melhor equação.

Leite et al. (2011) avaliando seis modelos volumétricos para Eucalyptus urophylla implantado no espaçamento 3,0 $\mathrm{m}$ x 2,0 $\mathrm{m}$ em Mineiros, GO verificaram melhor estimativa volumétrica com a equação de Schumacher-Hall. 
Tabela 5. Parâmetros estimados e medidas de precisão das equações volumétricas ajustadas para o híbrido E. grandis $\mathrm{x}$ E. camaldulensis (clone 1277), implantado no espaçamento 3,0 m x 1,5 m, em Jataí-GO.

\begin{tabular}{cccccccc}
\hline Equação & $\beta_{0}$ & $\beta_{1}$ & $\beta_{2}$ & $\beta_{3}$ & $\mathrm{R}^{2}$ aj. $(\%)$ & $\mathrm{S}_{\mathrm{yx}}(\%)$ & $\mathrm{F}$ \\
\hline Hohenadl-Krenn & $-0,01394$ & 0,00192 & 0,000458 & & 92,8 & $\pm 6,9$ & 7,6 \\
Husch & $-8,01854$ & 2,18481 & & & 94,1 & $\pm 6,9$ & 6,3 \\
Brenac & $-5,81875$ & $-6,63212$ & 1,52107 & & 94,2 & $\pm 6,9$ & 7,4 \\
Spurr* & 0,00743 & 0,00003 & & & 94,1 & $\pm 6,1$ & 12,2 \\
Stoate & $-0,00082$ & 0,00026 & 0,00001 & 0,00015 & 95,8 & $\pm 5,3$ & 12,7 \\
Schumacher-Hall & 0,00015 & 1,96781 & 0,50701 & & 95,8 & $\pm 5,1$ & 19,8
\end{tabular}

Em que: $\beta_{0}, \beta_{1}, \beta_{2} \beta_{3}=$ coeficientes da regressão, $\mathrm{R}^{2}$ aj. $(\%)=$ coeficiente de determinação ajustado, $\mathrm{S}_{\mathrm{yx}}(\%)=$ erro em porcentagem, $\mathrm{F}$ $=$ estatística $\mathrm{F}$ de Fischer, $*=$ modelo selecionado.

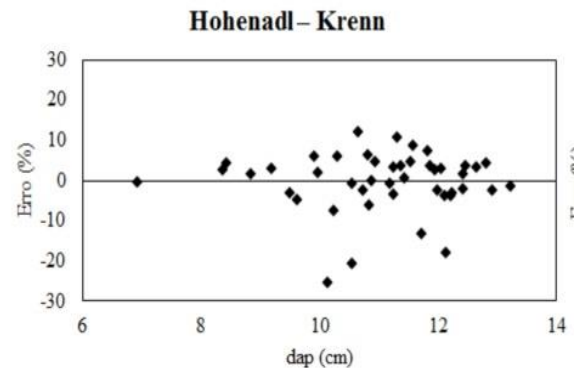

Spurr
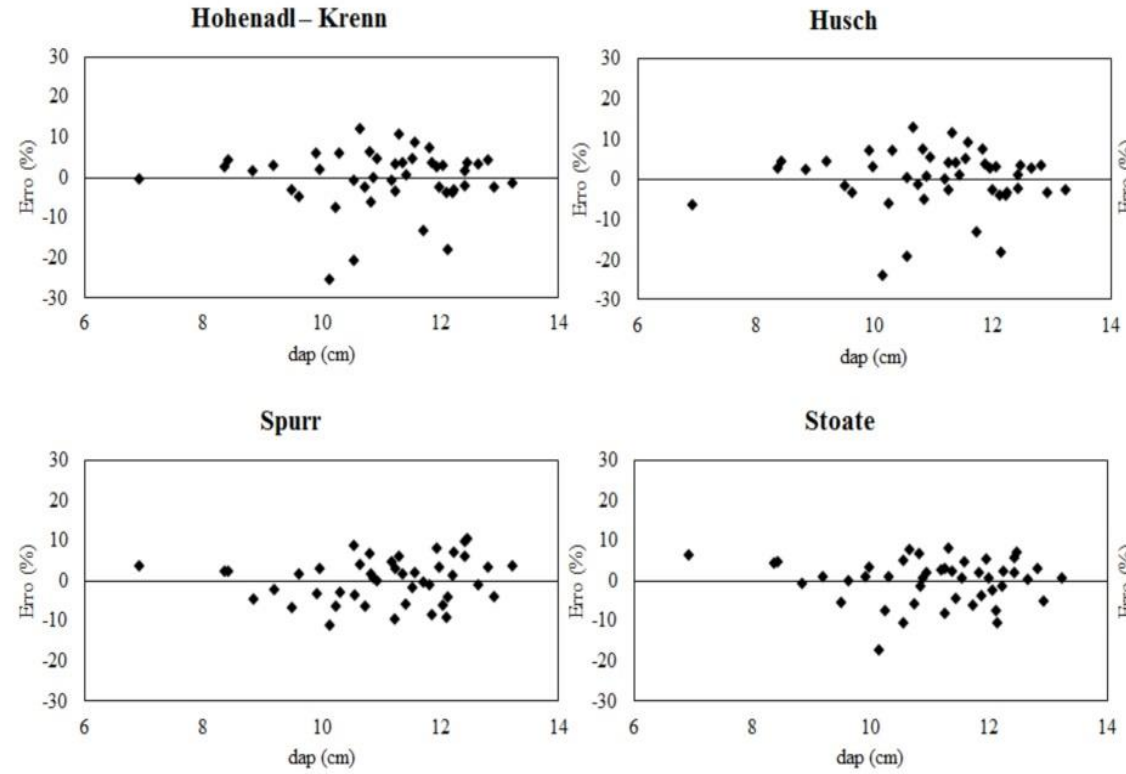

Stoate
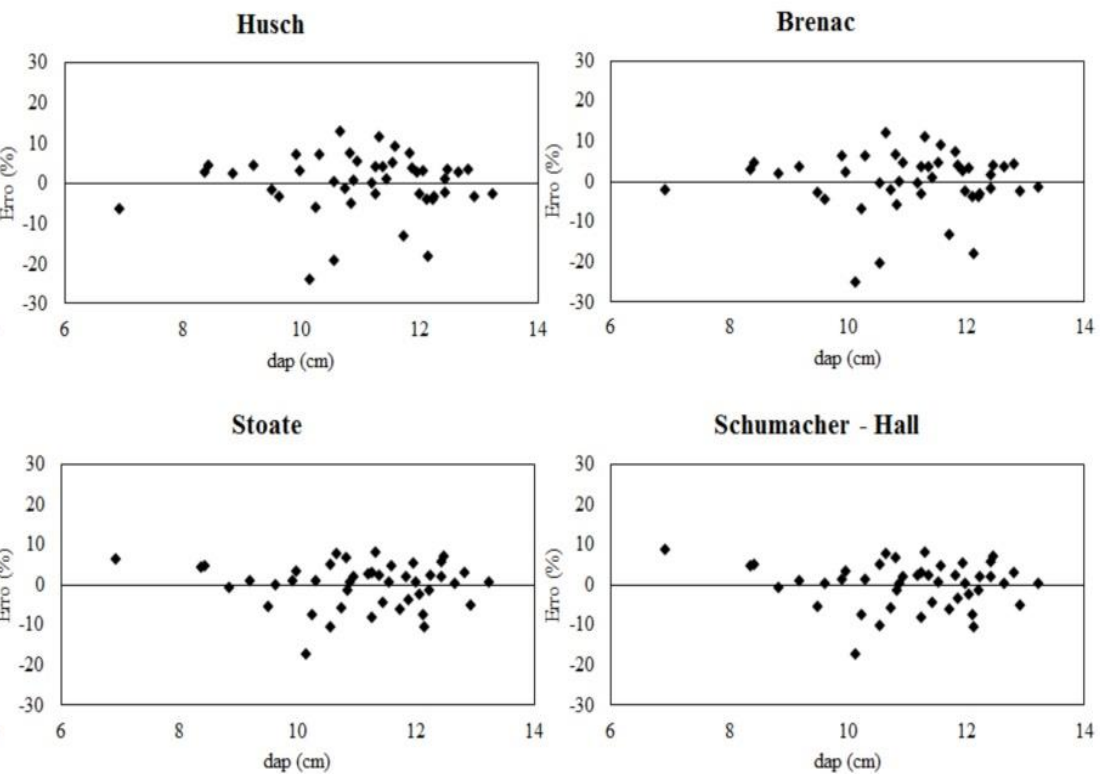

Schumacher - Hall

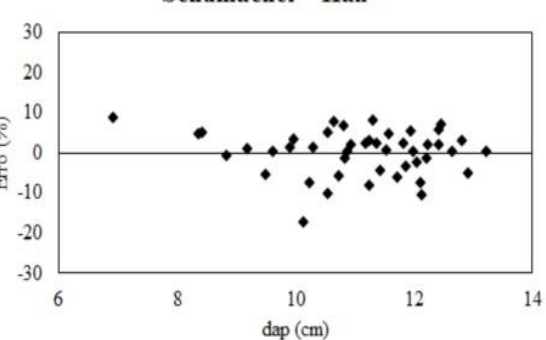

Figura 3. Resíduos em função do diâmetro para as equações volumétricas ajustadas para o híbrido E. grandis x E. camaldulensis (clone 1277) implantado no espaçamento 3,0 m x 1,5 m em Jataí-GO.
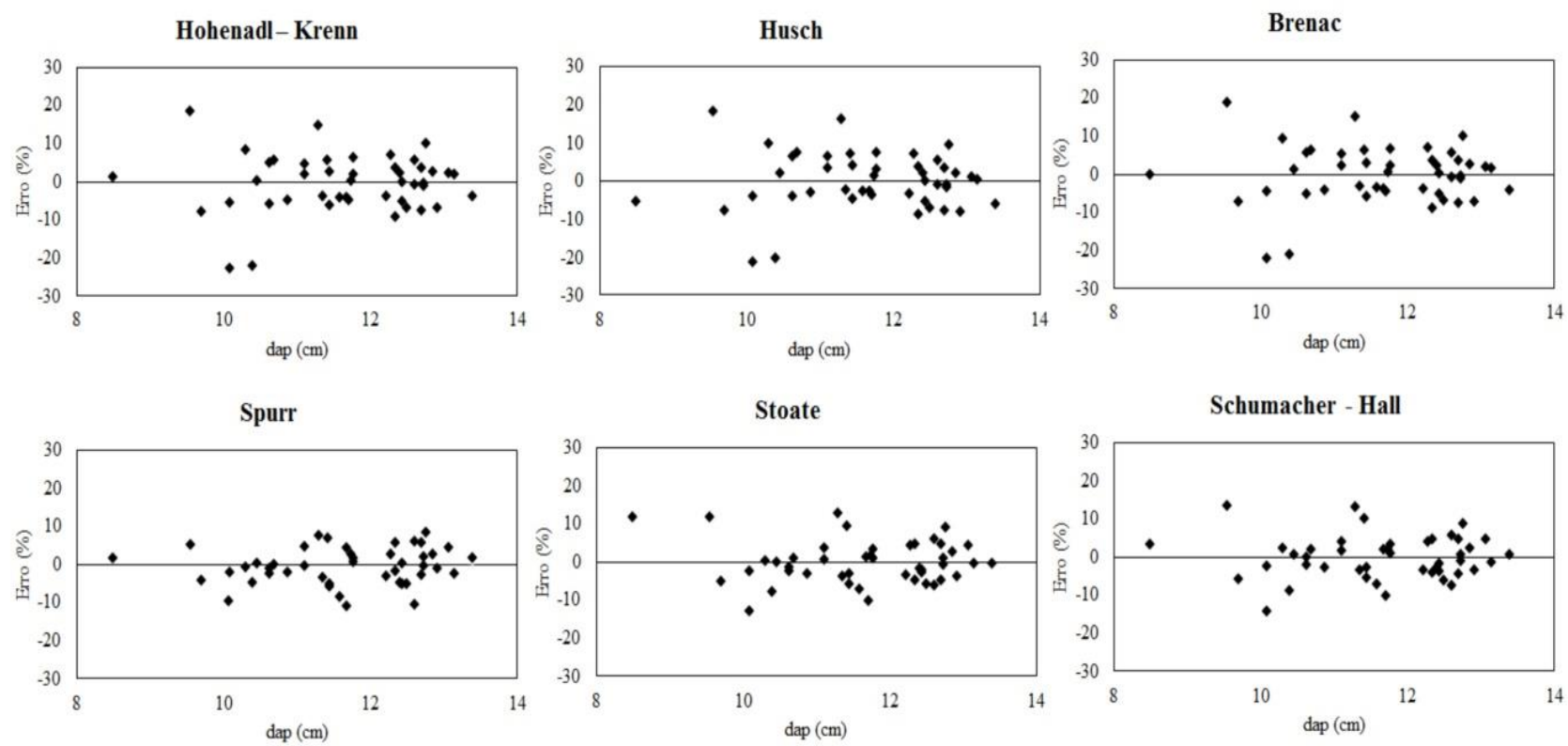

Figura 4. Resíduos em função do diâmetro para as equações volumétricas ajustadas para o híbrido E. grandis $\mathrm{x}$ E. camaldulensis (clone 1277) implantado no espaçamento 3,0 m x 2,0 m em Jataí-GO. 
Tabela 6. Parâmetros estimados e medidas de precisão das equações volumétricas ajustadas para o híbrido $E$. grandis x $E$ camaldulensis (clone 1277), implantado no espaçamento 3,0 m x 2,0 m, em Jataí-GO.

\begin{tabular}{cccccccc}
\hline Equação & $\beta_{0}$ & $\beta_{1}$ & $\beta_{2}$ & $\beta_{3}$ & $\mathrm{R}^{2}$ aj. $(\%)$ & $\mathrm{S}_{\mathrm{yx}}(\%)$ & $\mathrm{F}$ \\
\hline Hohenadl-Krenn & $-0,06180$ & 0,00005 & 0,01090 & & 88,6 & $\pm 6,7$ & 16,7 \\
Husch & $-7,75558$ & 2,08496 & & & 88,2 & $\pm 6,7$ & 15,0 \\
Brenac & $-2,2314$ & 0,4640 & $-17,9046$ & & 88,5 & $\pm 6,7$ & 25,7 \\
Spurr* & 0,01010 & 0,00004 & & & 91,5 & $\pm 5,8$ & 31,1 \\
Stoate & $-0,03917$ & 0,00052 & $-0,000004$ & 0,00360 & 92,1 & $\pm 5,8$ & 9,9 \\
Schumacher-Hall & 0,00018 & 1,82881 & 0,58941 & & 92,4 & $\pm 5,5$ & 18,9 \\
\hline
\end{tabular}

Em que: $\beta_{0}, \beta_{1}, \beta_{2} \beta_{3}=$ coeficientes da regressão, $\mathrm{R}^{2}$ aj. $(\%)=$ coeficiente de determinação ajustado, $\mathrm{S}_{\mathrm{yx}}(\%)=$ erro em porcentagem, $\mathrm{F}=$ estatística $\mathrm{F}$ de Fischer, $*=$ modelo selecionado.

Tabela 7. Parâmetros estimados e medidas de precisão das equações volumétricas ajustadas para o híbrido E. grandis x E. camaldulensis (clone 1277), implantado no espaçamento 3,0 m x 2,5 m, em Jataí-GO.

\begin{tabular}{cccccccc}
\hline Equação & $\beta_{0}$ & $\beta_{1}$ & $\beta_{2}$ & $\beta_{3}$ & $\mathrm{R}^{2}$ aj. $(\%)$ & $\mathrm{S}_{\mathrm{yx}}(\%)$ & $\mathrm{F}$ \\
\hline Hohenadl-Krenn & $-0,11943$ & $-0,00041$ & 0,02131 & & 91,3 & $\pm 5,2$ & 12,0 \\
Husch & $-7,06773$ & 1,80807 & & 90,9 & $\pm 5,4$ & 16,0 \\
Brenac & 5,3221 & $-1,7422$ & $-42,5965$ & & 91,9 & $\pm 5,3$ & 27,6 \\
Spurr & 0,02643 & 0,00003 & & & 91,9 & $\pm 4,9$ & 9,58 \\
Stoate* & $-0,05969$ & 0,00064 & $-0,00002$ & 0,00605 & 93,8 & $\pm 4,3$ & 15,3 \\
Schumacher-Hall & 0,00058 & 1,49086 & 0,45409 & & 93,9 & $\pm 4,4$ & 13,4 \\
\hline
\end{tabular}

Em que: $\beta_{0}, \beta_{1}, \beta_{2} \beta_{3}=$ coeficientes da regressão, $\mathrm{R}^{2}$ aj. $(\%)=$ coeficiente de determinação ajustado, $\mathrm{S}_{\mathrm{yx}}(\%)=$ erro em porcentagem, $\mathrm{F}=$ estatística $\mathrm{F}$ de Fischer, $*$ modelo selecionado.
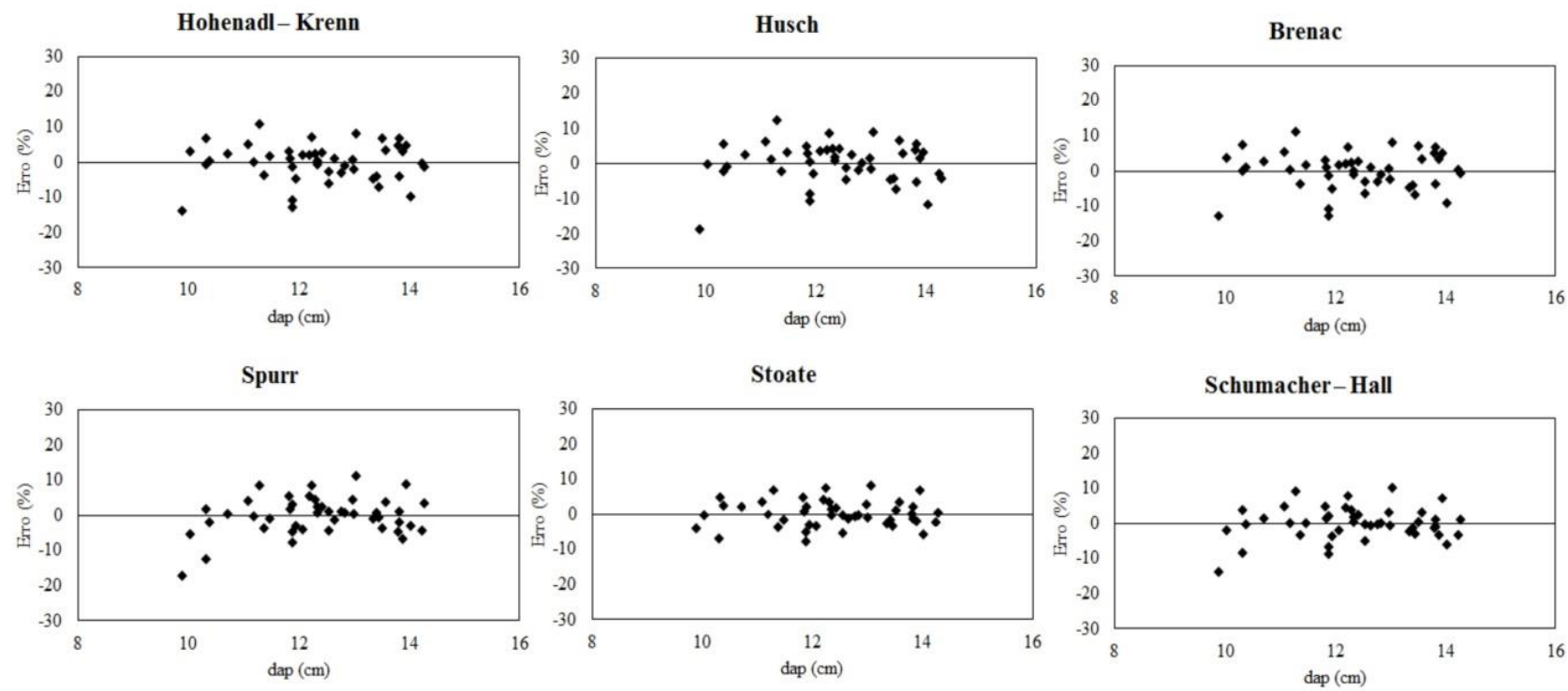

Figura 5. Resíduos em função do diâmetro para as equações volumétricas ajustadas para o híbrido E. grandis x E. camaldulensis (clone 1277) implantado no espaçamento 3,0 m x 2,5 m em Jataí-GO.

Verificou-se que, em geral, os ajustes realizados obtiveram bons resultados com o coeficiente de determinação variando entre $79,4 \%$ e $98,9 \%$ e o erro padrão da estimativa variando entre $3,6 \%$ e $6,9 \%$.

As equações de dupla entrada, ou seja, as com duas variáveis dependentes (dap e HT), apresentaram melhores ajustes com relação às equações de simples entrada. Este resultado se encontra dentro do esperado, uma vez que a altura responde por grande parte da variação em volume de uma árvore. Autores como Schröder et al. (2013) e Pereira et al. (2016), também obtiveram maior precisão em equações de dupla entrada para estimativas volumétricas de povoamentos de eucalipto.

\section{Teste de identidade - Modelo de Spurr}

Para certas combinações o modelo reduzido não pode ser utilizado na estimativa volumétrica das árvores. A equação volumétrica do modelo reduzido a ser utilizada para os espaçamentos combinados é $3,0 \mathrm{~m}$ x $0,5 \mathrm{~m} \mathrm{e} 3,0 \mathrm{~m}$ x $2,0 \mathrm{~m}$ é $V=0,006661+$ $0,000036 *$ dap $^{2 *} h$ com $\mathrm{R}^{2}=97,1 \%$ e $\mathrm{S}_{\mathrm{yx}}= \pm 5,9 \%$, cujo gráfico de distribuição dos resíduos é apresentado na Figura 7. 
Tabela 8. Parâmetros estimados e medidas de precisão das equações volumétricas ajustadas para o híbrido $E$. grandis x $E$. camaldulensis (clone 1277) implantado no espaçamento 3,0 m x 3,0 m em Jataí-GO.

\begin{tabular}{cccccccc}
\hline Equação & $\beta_{0}$ & $\beta_{1}$ & $\beta_{2}$ & $\beta_{3}$ & $\mathrm{R}^{2}$ aj. $(\%)$ & $\mathrm{S}_{\mathrm{yx}}(\%)$ & $\mathrm{F}$ \\
\hline Hohenadl-Krenn & $-0,00909$ & 0,00176 & 0,00045 & & 90,6 & $\pm 6,8$ & 16,3 \\
Husch & $-7,48902$ & 1,97893 & & & 89,1 & $\pm 6,7$ & 20,8 \\
Brenac & $-6,19311$ & 1,60887 & $-4,48997$ & & 89,1 & $\pm 6,8$ & 21,3 \\
Spurr & 0,00562 & 0,00004 & & & 79,4 & $\pm 6,3$ & 21,5 \\
Stoate & $-0,01975$ & 0,00048 & 0,000005 & 0,00117 & 93,8 & $\pm 5,4$ & 17,8 \\
Schumacher-Hall* $^{*}$ & $-0,11095$ & 0,01360 & 0,00176 & & 93,4 & $\pm 5,6$ & 19,9 \\
\hline
\end{tabular}

Em que: $\beta_{0}, \beta_{1}, \beta_{2} \beta_{3}=$ coeficientes da regressão, $\mathrm{R}^{2}$ aj. $(\%)=$ coeficiente de determinação ajustado, $\mathrm{S}_{\mathrm{yx}}(\%)=$ erro em porcentagem, $\mathrm{F}=$ estatística $\mathrm{F}$ de Fischer, $*=$ modelo selecionado.
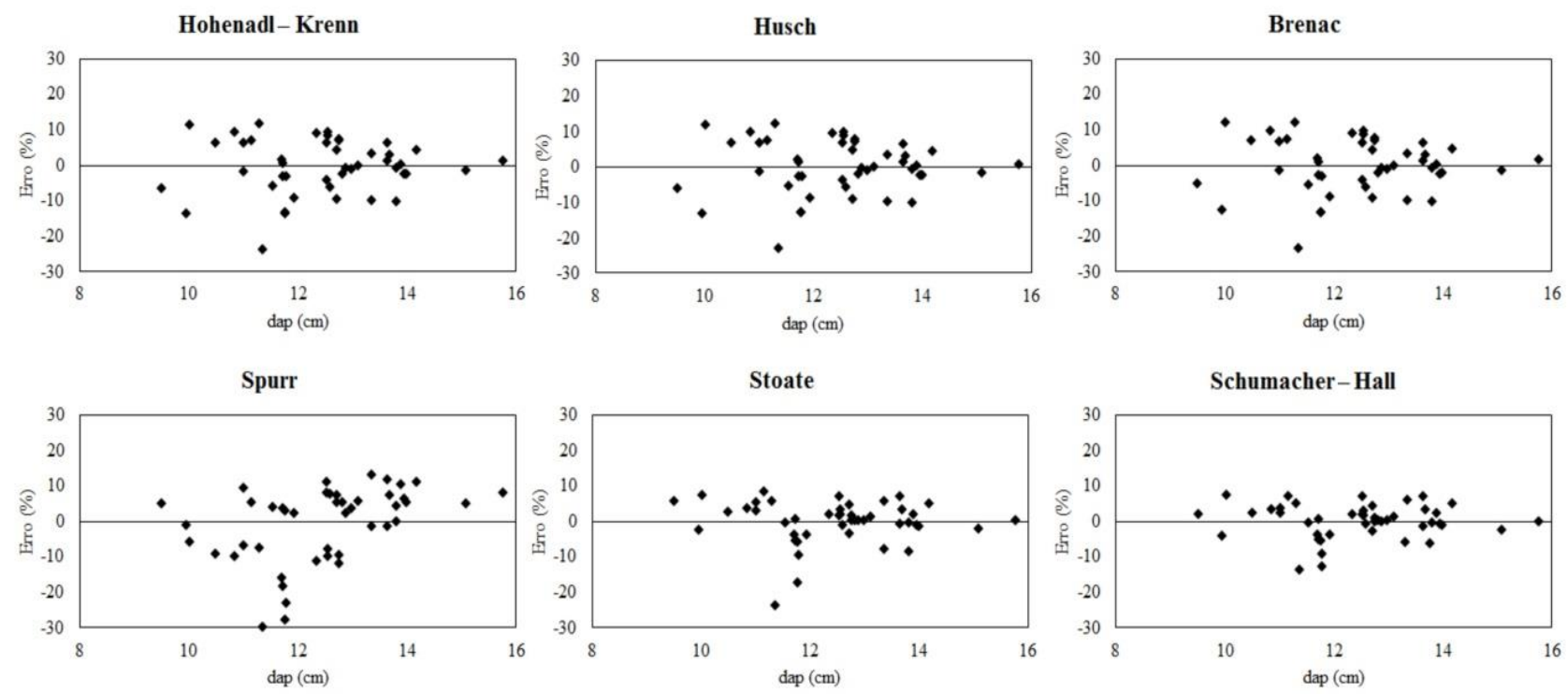

Figura 6. Resíduos em função do diâmetro para as equações volumétricas ajustadas para o híbrido E. grandis x E. camaldulensis (clone 1277) implantado no espaçamento 3,0 m x 3,0 m em Jataí-GO.

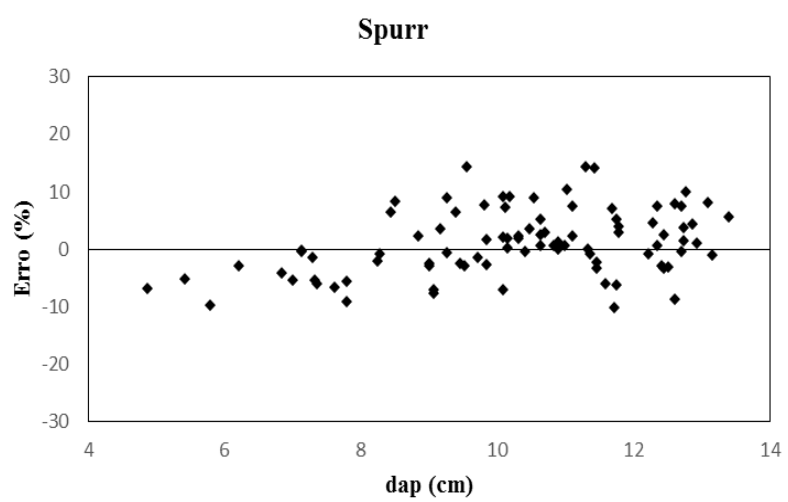

Figura 7. Resíduos em função do diâmetro para a equação volumétrica ajustada com os espaçamentos combinados.

A análise de variância para testar a identidade dos modelos de regressão é apresentada na Tabela 9.

Somente nas combinações dos espaçamentos $3,0 \mathrm{~m}$ x $0,5 \mathrm{~m} \mathrm{e} 3,0 \mathrm{~m} \mathrm{x} 2,0 \mathrm{~m}$ o valor de $f_{\text {calculado }} 1,19$ foi menor que o valor de $f_{\text {tabelado, }}$, verificando-se assim que não existe diferença a nível de $5 \%$ de significância entre o modelo completo e o reduzido para esta combinação. Neste caso, pode-se empregar o modelo reduzido para a estimativa volumétrica de árvores individuais.

\section{Modelo de Schumacher-Hall}

O teste qui-quadrado para o modelo de SchumacherHall na estimativa do volume de árvores foi significativo $\left(\mathrm{X}^{2}\right.$ calculado $=14,15^{*}$ e $\mathrm{X}^{2}$ tabelado $=$ 7,81 ). Desta forma, para os espaçamentos $3,0 \mathrm{~m} \mathrm{x} \mathrm{1,0} \mathrm{m}$ e 3,0 m x 2,0 m rejeita-se a hipótese $\mathrm{H} 0$, ou seja, a um nível de 5\% de significância não é permitido utilizar o modelo reduzido para estimar o volume das árvores de Eucalyptus. O que implica que para o modelo de Schumacher-Hall, faz-se necessária a utilização de uma equação para cada um desses espaçamentos.

\section{Fator de forma}

Os fatores de forma obtidos para os diferentes espaçamentos estudados estão apresentados na Tabela 10. A variação do fator de forma ficou entre 0,48 e 0,56. Espaçamentos menores tendem a ter árvores mais cilíndricas refletindo em fatores de forma maiores. Van Laar (1978), trabalhando com Pinus patula, verificou que o fator de forma diminui com o aumento do espaçamento, sendo este 0,47 quando haviam 902 arvores/ha e 0,44 quando haviam 124 árvores/ha. Figueiredo et al. (2005) verificaram para teca (Tectona 
grandis L.f.) que árvores mais jovens são normalmente mais cilíndricas e então possuem fatores de forma maiores e árvores mais cônicas geralmente mais velhas possuem fatores de forma menores. Cabacinha et al. (2003) obtiveram fatores de forma para Eucalyptus em densidade de plantio $2,5 \mathrm{~m} \times 2,5 \mathrm{~m}$, na idade de 6,5 anos, variando entre 0,43 e 0,52 para toretes de 2,0 m. Drescher et al. (2010) encontraram valores entre 0,40 e 0,64 para povoamentos de Tectona grandis implantados no espaçamento 3,0 $\mathrm{m}$ x 2,0 $\mathrm{m}$ aos 6 anos de idade.

Tabela 10. Fatores de forma para todos os espaçamentos do híbrido E. grandis x E. camaldulensis(clone 1277) implantado em Jataí-GO.

\begin{tabular}{cc}
\hline Espaçamento & Fatores de Forma \\
\hline $3,0 \mathrm{~m} \times 0,5 \mathrm{~m}$ & 0,56 \\
$3,0 \mathrm{~m} \times 1,0 \mathrm{~m}$ & 0,51 \\
$3,0 \mathrm{~m} \times 1,5 \mathrm{~m}$ & 0,50 \\
$3,0 \mathrm{~m} \times 2,0 \mathrm{~m}$ & 0,52 \\
$3,0 \mathrm{~m} \times 2,5 \mathrm{~m}$ & 0,49 \\
$3,0 \mathrm{~m} \times 3,0 \mathrm{~m}$ & 0,48 \\
\hline Valor Médio & 0,51 \\
\hline
\end{tabular}

\section{Fatores de empilhamento e cubicação}

$\mathrm{Na}$ Tabela 11 estão os resultados encontrados para os fatores de empilhamento e cubicação para cada espaçamento estudado. Os fatores de empilhamento variaram entre 1,44 e 2,05 e os fatores de cubicação variaram de 0,57 a 0,70. Verificou-se que os maiores espaçamentos apresentam menores valores de fator de empilhamento e os menores espaçamentos, os quais possuem árvores de menores diâmetros, apresentam maiores valores para o fator de empilhamento.

Tabela 11. Fatores de cubicação e empilhamento para todos os espaçamentos para o híbrido E. grandis x E. camaldulensis (clone 1277) implantado em Jataí-GO.

\begin{tabular}{ccc}
\hline Espaçamentos & $\begin{array}{c}\text { Fator de } \\
\text { Empilhamento }\end{array}$ & $\begin{array}{c}\text { Fator de } \\
\text { Cubicação }\end{array}$ \\
\hline $3,0 \mathrm{~m} \times 0,5 \mathrm{~m}$ & 1,77 & 0,57 \\
$3,0 \mathrm{~m} \times 1,0 \mathrm{~m}$ & 1,75 & 0,58 \\
$3,0 \mathrm{~m} \times 1,5 \mathrm{~m}$ & 2,05 & 0,50 \\
$3,0 \mathrm{~m} \times 2,0 \mathrm{~m}$ & 1,65 & 0,61 \\
$3,0 \mathrm{~m} \times 2,5 \mathrm{~m}$ & 1,62 & 0,62 \\
$3,0 \mathrm{~m} \times 3,0 \mathrm{~m}$ & 1,44 & 0,70 \\
\hline Valor Médio & 1,71 & 0,60 \\
\hline
\end{tabular}

Paula Neto et al. (1993) relatam que os maiores diâmetros das toras geram menores fatores de empilhamento. Ainda de acordo com os autores, quanto maior a classe de dap, maiores volumes de madeira em estere serão necessários para completar um metro cúbico de madeira sólida, em virtude da maior quantidade de espaços vazios na pilha, acarretando em fatores de cubicação menores.

Barros et al. (2008), estudando fatores de cubicação para toretes de Eucalyptus grandis oriundo de um povoamento com 11 anos de idade, obtiveram valores entre 0,64 e 0,71 para pilhas formadas no verão com toretes de comprimentos variando entre $1,0 \mathrm{~m}$ e 2,3 $\mathrm{m}$.

Tabela 9. Análise de variância para o teste de identidade do modelo de Spurr.

\begin{tabular}{|c|c|c|c|c|c|}
\hline Combinações & FV & GL & SQ & QM & $\mathrm{f}_{\text {calc. }}$ \\
\hline \multirow{5}{*}{$3,0 \mathrm{~m} \times 0,5 \mathrm{~m}+3,0 \mathrm{~m} \times 1,5 \mathrm{~m}+3,0 \mathrm{~m} \times 2,0 \mathrm{~m}$} & Modelo completo & 6 & 0,547667 & & \\
\hline & Modelo reduzido & 2 & 0,547234 & & \\
\hline & Diferença & 4 & 0,000432 & 0,00011 & \\
\hline & Resíduo & 129 & 0,001731 & 0,00001 & $8,05^{*}$ \\
\hline & Total & 135 & 0,549397 & & \\
\hline \multirow{5}{*}{$3,0 \mathrm{~m} \times 0,5 \mathrm{~m}+3,0 \mathrm{~m} \times 1,5 \mathrm{~m}$} & Modelo completo & 4 & 0,301900 & & \\
\hline & Modelo reduzido & 2 & 0,301749 & & \\
\hline & Diferença & 2 & 0,000151 & 0,00008 & \\
\hline & Resíduo & 86 & 0,000965 & 0,00001 & $6,72 *$ \\
\hline & Total & 90 & 0,302865 & & \\
\hline \multirow{5}{*}{$3,0 \mathrm{~m} \times 0,5 \mathrm{~m}+3,0 \mathrm{~m} \times 2,0 \mathrm{~m}$} & Modelo completo & 4 & 0,351517 & & \\
\hline & Modelo reduzido & 2 & 0,351488 & & \\
\hline & Diferença & 2 & 0,000029 & 0,00001 & \\
\hline & Resíduo & 86 & 0,001051 & 0,00001 & $1,19 \mathrm{~ns}$ \\
\hline & Total & 90 & 0,352569 & & \\
\hline \multirow{5}{*}{$3,0 \mathrm{~m} \times 1,5 \mathrm{~m}+3,0 \mathrm{~m} \times 2,0 \mathrm{~m}$} & Modelo completo & 4 & 0,441916 & & \\
\hline & Modelo reduzido & 2 & 0,441517 & & \\
\hline & Diferença & 2 & 0,000399 & 0,00020 & \\
\hline & Resíduo & 86 & 0,001446 & 0,00002 & $11,85^{*}$ \\
\hline & Total & 90 & 0,443362 & & \\
\hline
\end{tabular}




\section{Conclusões}

A equação oriunda do modelo de Spurr foi selecionada para os espaçamentos $3,0 \mathrm{~m}$ x $0,5 \mathrm{~m} ; 3,0 \mathrm{~m}$ x 1,5 m e 3,0 m x 2,0 m. A equação originada pelo modelo de Schumacher-Hall foi selecionada para os espaçamentos $3,0 \mathrm{~m}$ x $1,0 \mathrm{~m}$ e $3,0 \mathrm{~m} \times 3,0 \mathrm{~m}$ e a equação gerada pelo modelo de Stoate foi selecionada para o espaçamento $3,0 \mathrm{~m} \times 2,5 \mathrm{~m}$.

Pelo teste de identidade de modelos, verificou-se que apenas nas junções dos espaçamentos $3,0 \mathrm{~m}$ x 0,5 m e 3,0 $\mathrm{m}$ x 2,0 m pode-se utilizar um modelo reduzido.

Os fatores de forma variaram entre 0,48 e 0,56 , enquanto que os fatores de empilhamento entre 1,44 a 2,05 e os fatores de cubicação de 0,57 a 0,70 .

\section{Referências Bibliográficas}

AZEVEDO, G. B.; SOUSA, G. T. O.; BARRETO, P. A. B.; CONCEIÇÃO JÚNIOR, V. Estimativas volumétricas em povoamentos de eucalipto sob regime de alto fuste e talhadia no sudoeste da Bahia. Pesquisa Florestal Brasileira, Colombo-PR, v. 31, n. 68, p. 309-318, 2011.

BARROS, M. V.; FINGER, C. A. G.; SCHNEIDER, P. R.; SANTINI, E. J. Fator de cubicação para toretes de Eucalyptus grandis e sua variação com o tempo de exposição ao ambiente. Revista Ciência Florestal, Santa Maria-RS, v. 18, n. 3, p. 109-119, 2008.

CABACINHA, C. D.; MELlO, J. M.; DALANESI, P. E. Acuracidade nas estimativas volumétricas de Eucalyptus saligna, Revista Científica Eletrônica de Engenharia Florestal, Garça-SP, v. 1, n. 2, p. 1-4, 2003.

CAMOLESI, J. F; SCOLFORO, J. R. S.; OLIVEIRA, A. D.; ACERBI JÚNIOR, F. W.; RUFINI, A. L.; MELLO, J. M. Ajuste, seleção e teste de identidade de modelo para volume e número de moirões da candeia (Eremanthus erythropappus). Cerne, Lavras-MG, v. 16, n. 4, p. 431-441, 2010.

DRESCHER, R.; PELISSARI, A. L.; GAVA, F. H. Fator de forma artificial para povoamentos jovens de Tectona grandis em Mato Grosso. Revista Pesquisa Florestal Brasileira, Colombo-PR, v. 30, n. 63, p. 1-7, 2010.

FIGUEIREDO, E. O.; SCOLFORO, J. R. S.; OLIVEIRA, A. D. Estimativa de percentual de casca e do fator de forma em povoamentos jovens de teca (Tectona grandis L.f.). Rio Branco-AC: Embrapa Acre, 2005. 5 p. (Comunicado Técnico, 165)

GRAYBILL, F. A. Theory and application of the linear model. Belmont: Duxbury Press; 1976. 704 p.

KURCHAIDT, S. M.; ZANETTE, V. H.; KOEHLER, H. S. Identidade de modelos em fragmento de floresta ombrófila mista. Enciclopédia Biosfera, Goiânia-GO, v. 10, n. 19, p. 2302-2311, 2014.

LEITE, D. R.; MIGUEL, E. P; SANTOS, G. A.; ENCINAS, M. I.; REZENDE, A. V. Análise comparativa entre dois procedimentos para estimativa da variável volume em um povoamento de Eucalyptus urophylla, no município de Mineiros - Goiás. Enciclopédia Biosfera, Goiânia-GO, v. 7, n. 13, p. 1684-1700, 2011.
MELO, L. C.; BARRETO, P. A. B.; OLIVEIRA, F. G. R. B.; NOVAES, A. B. Estimativas volumétricas em povoamentos de Pinus caribaeae var. hondurensis no sudoeste da Bahia. Revista Pesquisa Florestal Brasileira; Colombo-PR, v. 33, n. 76, p.1-8, 2013.

MIGUEL, E. P.; CANZI, L. F.; RUFINO, R. F.; SANTOS, G. A. Ajuste de modelo volumétrico e desenvolvimento de fator de forma para plantios de Eucalyptus grandis localizados no município de Rio Verde - GO. Revista Enciclopédia Biosfera, Goiânia-GO, v. 6, n. 11, p. 1-13, 2010.

NICOLETTI, M. F.; SOUZA, K.; SILVESTRE, R.; FRANÇA, M. C.; ROLIM, F. A. Relação hipsométrica para Pinus taeda L. em diferentes fases do ciclo de corte. Floresta e Ambiente, Seropédica-RJ, v. 23, n. 1, p. 80-89, 2016.

PACHECO J. M.; FIGUEIREDO FILHO A. F.; DIAS N. A.; MACHADO A. S.; LIMA R. Efeito da densidade inicial no crescimento de Pinus taeda L. na região Centro Sul do Paraná. Scientia Forestalis, Piracicaba-SP, v. 43, n. 106, p. 353-365, 2015.

PAULA NETO, F.; REZENDE, A. V.; CAMPOS, J. C. C.; REZENDE, J. L. P. Análise do comportamento dos fatores de empilhamento para Eucalyptus grandis. Revista Árvore, Viçosa-MG, v. 17, n. 1, p. 45-59, 1993.

PEREIRA, A. R. S.; CORDEIRO, M. A.; ABREU, J. C.; SANTOS, R. O.; SILVA, J. N. M. Modelagem volumétrica para Eucalyptus urograndis no município de Porto Grande, Amapá, Brasil. Biota Amazônia, Macapá-AM, v. 6 n. 4, p. 10-14, 2016

QUEIROZ, D.; MACHADO, A. S.; FIGUEIREDO FILHO, A.; ARCE, J. E.; KOEHLER, H. S. Identidade de modelos em funções de afilamento para Mimosa scabrella Bentham em povoamentos nativos da região metropolitana de Curitiba/PR. Floresta, Curitiba-PR, v. 38, n. 2, p. 339-349, 2008.

REGAZZI, A. J.; SILVA, C. H. O. Testes para verificar a igualdade de parâmetros e a identidade de modelos de regressão não-linear em dados de experimento com delineamento em blocos casualizados. Revista Ceres, ViçosaMG, v. 57, n. 3, p. 315-320, 2010.

ROCHA T. B.; CABACINHA C. D.; ALMEIDA R. C.; PAULA A.; SANTOS R. C. Avaliação de métodos de estimativa de volume para um povoamento de Eucalyptus urophylla S. T. Blake no Planalto da Conquista-BA. Enciclopédia Biosfera, Goiânia-GO, v. 6, n. 10, p. 1-13, 2010 .

SCHRÖDER T.; PEREIRA L. D.; HOFIÇO N. S. A. Comparação de métodos de estimativa de volume total para Eucalyptus grandis W. Hill ex Maiden. Floresta e Ambiente, Seropédica-RJ, v. 20, n. 4, p. 480-486, 2013.

THOMAS, C.; ANDRADE, C. M; SCHNEIDER, P. R.; FINGER, C. A. G. Comparação de equações volumétricas ajustadas com dados de cubagem e análise de tronco. Revista Ciência Florestal, Santa Maria-RS, v. 16, n. 3, p. 319-327, 2006.

VAN LAAR, A. The growth of unthinned Pinus patula in relation to spacing. South African Forestry Journal, Stellenbosch, n. 107, p. 3-11, 1978. 